\title{
Nationwide cloud-based integrated database of idiopathic interstitial pneumonias for multidisciplinary discussion
}

Tomoyuki Fujisawa ${ }^{1}$, Kazutaka Mori ${ }^{1}$, Masashi Mikamo', Takashi Ohno², Kensuke Kataoka ${ }^{3}$, Chikatoshi Sugimoto ${ }^{4}$, Hideya Kitamura ${ }^{5}$, Noriyuki Enomoto ${ }^{1}$, Ryoko Egashira ${ }^{6}$, Hiromitsu Sumikawa ${ }^{7},{ }^{1}$ Tae Iwasawa ${ }^{8}$, Shoichiro Matsushita?, Hiroaki Sugiura ${ }^{10}$, Mikiko Hashisako ${ }^{11}$, Tomonori Tanaka ${ }^{12}$, Yasuhiro Terasaki ${ }^{13}$, Shinobu Kunugi ${ }^{13}$, Masashi Kitani ${ }^{14}$, Ryo Okuda ${ }^{5}$, Yasuoki Horiike ${ }^{1}$, Yasunori Enomoto ${ }^{1}$, Hideki Yasui ${ }^{1}$, Hironao Hozumi ${ }^{1}$, Yuzo Suzuki ${ }^{1}$, Yutaro Nakamura ${ }^{1}$, Junya Fukuoka ${ }^{15}$, Takeshi Johkoh ${ }^{16}$, Yasuhiro Kondoh ${ }^{3}$, Takashi Ogura ${ }^{5}$, Yoshikazu Inoue ${ }^{4}$, Yoshinori Hasegawa ${ }^{17}$, Naohiko Inase ${ }^{18}$, Sakae Homma ${ }^{19}$ and Takafumi Suda ${ }^{1}$

@ERSpublications

A cloud-based integrated database of idiopathic interstitial pneumonias containing clinical, radiological and pathological data along with a web-based multidisciplinary discussion system can make discussions more feasible and improve disease management http://ow.ly/NqqD30nYenb

Cite this article as: Fujisawa T, Mori K, Mikamo M, et al. Nationwide cloud-based integrated database of idiopathic interstitial pneumonias for multidisciplinary discussion. Eur Respir J 2019; 53: 1802243 [https:// doi.org/10.1183/13993003.02243-2018].

ABSTRACT Multidisciplinary discussion (MDD) requiring close communication between specialists (clinicians, radiologists and pathologists) is the gold standard for the diagnosis of idiopathic interstitial pneumonias (IIPs). However, MDD by specialists is not always feasible because they are often separated by time and location. An online database would facilitate data sharing and MDD. Our aims were to develop a nationwide cloud-based integrated database containing clinical, radiological and pathological data of patients with IIPs along with a web-based MDD system, and to validate the diagnostic utility of web-based MDD in IIPs.

Clinical data, high-resolution computed tomography images and lung biopsy slides from patients with IIPs were digitised and uploaded to separate servers to develop a cloud-based integrated database. Web-based MDD was performed using the database and video-conferencing to reach a diagnosis.

Clinical, radiological and pathological data of 524 patients in 39 institutions were collected, uploaded and incorporated into the cloud-based integrated database. Subsequently, web-based MDDs with a pulmonologist, radiologist and pathologist using the database and video-conferencing were successfully performed for the 465 cases with adequate data. Overall, the web-based MDD changed the institutional diagnosis in 219 cases (47\%). Notably, the MDD diagnosis yielded better prognostic separation among the IIPs than did the institutional diagnosis.

This is the first study of developing a nationwide cloud-based integrated database containing clinical, radiological and pathological data for web-based MDD in patients with IIPs. The database and the webbased MDD system that we built made MDD more feasible in practice, potentially increasing accurate diagnosis of IIPs.

This article has supplementary material available from erj.ersjournals.com

Received: Nov 262018 | Accepted after revision: March 042019

Copyright $\odot$ ERS 2019. This article is open access and distributed under the terms of the Creative Commons Attribution Non-Commercial Licence 4.0. 


\section{Introduction}

Idiopathic interstitial pneumonias (IIPs) are a heterogeneous group of interstitial lung diseases (ILDs) of unknown aetiology. They are classified into distinct disease entities, such as idiopathic pulmonary fibrosis (IPF), idiopathic nonspecific interstitial pneumonia (iNSIP) and cryptogenic organising pneumonia (COP) [1-4]. Accurate diagnosis is essential for estimating prognosis and managing patients with IIPs [3, 5], including treatment selection (e.g. antifibrotic agents) [6-8].

Current guidelines emphasise multidisciplinary discussion (MDD) as the gold standard for the diagnosis of IIPs [2-4]. MDD has been proven to increase interobserver agreement among specialists [9, 10]. This indicates that clinicians become more confident of their diagnoses through this discussion. MDD requires close communication between clinicians, radiologists and pathologists. However, several situations preclude $\mathrm{MDD}$, such as a shortage of specialists in each field (e.g. thoracic radiologists and pulmonary pathologists), especially within the same institution. Indeed, there are very few institutions worldwide in which MDD diagnosis can be conducted internally. To consult with specialists located in other institutions, the radiological and pathological data (e.g. lung biopsy specimens) must be sent separately to each specialist for review, which is very time and cost consuming. Therefore, MDD is not always practical in routine clinical practice.

To address these issues and facilitate MDD in practice, a cloud-based database that integrates clinical, radiological and pathological data of patients with IIPs would be useful. Such a database system enables users (e.g. clinicians, radiologists and pathologists) to access and refer to all the data on the web, even if they are in different institutions, areas or countries. This would make it possible to achieve an MDD diagnosis on the internet without assembling specialists in each field in the same place.

The present study was conducted to develop a nationwide cloud-based integrated database with clinical, radiological and pathological data of patients with a diagnosis of IIPs along with a web-based MDD system. To validate the utility of MDD with this system, we performed web-based MDDs with specialists in pulmonology, radiology and pathology for 465 cases of biopsy-proven IIPs. Furthermore, we examined the system's diagnostic performance in terms of prognostic discrimination for IIP diagnoses.

\section{Methods}

Full details are available in the supplementary material.

\section{Subjects}

39 institutions certified by the Japanese Respiratory Society participated in the present study. The study assessed records of patients diagnosed with IIPs in those institutions who had undergone chest high-resolution computed tomography (HRCT) and surgical lung biopsy (SLB) from April 2009 to March 2014. This retrospective study was approved by the Institutional Review Board of the Hamamatsu University School of Medicine, Hamamatsu, Japan (approval E14-360) and the respective ethics committees of each participating institution.

\section{Collection of clinical, radiological and pathological data}

A case identification number was allocated to each patient for de-identification purposes. The patients' clinical and HRCT data within 3 months before the SLB were collected. The clinical data were gathered

Affiliations: 'Second Division, Dept of Internal Medicine, Hamamatsu University School of Medicine, Hamamatsu, Japan. ${ }^{2}$ Esite Healthcare Co., Ltd, Tokyo, Japan. ${ }^{3}$ Dept of Respiratory Medicine and Allergy, Tosei General Hospital, Seto, Japan. ${ }^{4}$ Clinical Research Center, National Hospital Organization Kinki-Chuo Chest Medical Center, Osaka, Japan. ${ }^{5}$ Dept of Respiratory Medicine, Kanagawa Cardiovascular and Respiratory Center, Yokohama, Japan. ${ }^{6}$ Dept of Radiology, Faculty of Medicine, Saga University, Saga, Japan. ${ }^{7}$ Dept of Radiology, Sakai City Medical Center, Sakai, Japan. ${ }^{8}$ Dept of Radiology, Kanagawa Cardiovascular and Respiratory Center, Yokohama, Japan. ${ }^{9}$ Dept of Radiology, St Marianna University School of Medicine, Kawasaki, Japan. ${ }^{10}$ Dept of Radiology, Keio University School of Medicine, Tokyo, Japan. ${ }^{11}$ Division of Diagnostic Pathology, Kyushu University Hospital, Fukuoka, Japan. ${ }^{12}$ Dept of Pathology, Kindai University Faculty of Medicine, Osaka, Japan. ${ }^{13}$ Dept of Analytic Human Pathology, Graduate School of Medicine, Nippon Medical School, Tokyo, Japan. ${ }^{14}$ Dept of Pathology, National Hospital Organization Tokyo National Hospital, Tokyo, Japan. ${ }^{15}$ Dept of Pathology, Nagasaki University Graduate School of Biomedical Sciences, Nagasaki, Japan. ${ }^{16}$ Dept of Radiology, Kinki Central Hospital of Mutual Aid Association of Public-School Teachers, Itami, Japan. ${ }^{17}$ Dept of Respiratory Medicine, Nagoya University Graduate School of Medicine, Nagoya, Japan. ${ }^{18}$ Dept of Respiratory Medicine, Tokyo Medical and Dental University, Tokyo, Japan. ${ }^{19}$ Dept of Respiratory Medicine, Toho University Omori Medical Center, Tokyo, Japan.

Correspondence: Tomoyuki Fujisawa, Second Division, Dept of Internal Medicine, Hamamatsu University School of Medicine, 1-20-1 Handayama Higashi-ku, Hamamatsu 431-3192, Japan.

E-mail: fujisawalahama-med.ac.jp 
from the medical records as text data files using commercially available software (FileMaker Platform; www.filemaker.com). HRCT images were obtained as DICOM (digital imaging and communications in medicine) files. Glass slides of lung biopsy specimens were digitised as whole-slide images using Philips Digital Pathology Solutions (Philips, Amsterdam, The Netherlands). The vital status of the patients was ascertained in October 2017 for survival analysis.

\section{Development of the cloud-based integrated database including clinical, radiological and pathological data}

An outline of the development process is shown in figure 1 . The web viewer system and data servers were provided by Esite Healthcare (Tokyo, Japan). The data centre included three servers, i.e. one each for clinical, radiological and pathological data. The respective data files were uploaded to each web server separately and the data on the separate servers were interlinked by the respective case identification numbers.

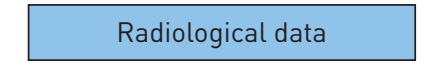

(DICOM files of chest HRCT)

Radiological data
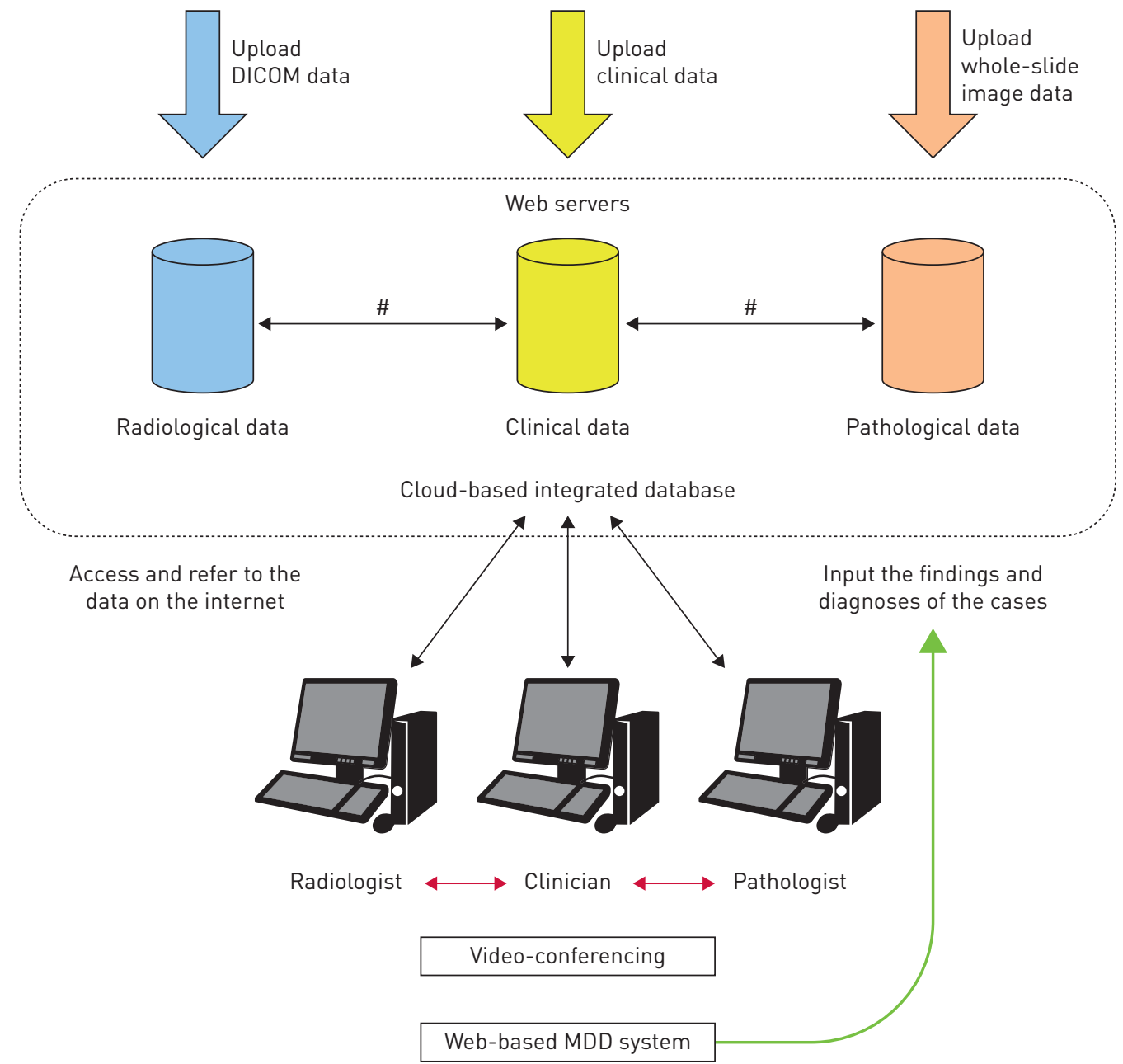

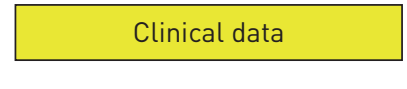

(Text data files of clinical data)

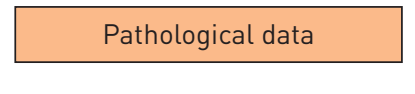

Whole-slide image data of surgical lung biopsyl

FIGURE 1 Schema of the development of the cloud-based integrated database including clinical radiological and pathological data, and web-based multidisciplinary discussion (MDD) using the cloud-based integrated database. HRCT: high-resolution computed tomography. The data centre included three servers, one each for clinical, radiological (HRCT DICOM files) and pathological (whole-slide files) data. Each type of data was uploaded to each web server separately and registered with the appropriate case identification numbers. For web-based MDD, the clinician, radiologist and pathologist referred to the data and evaluated the case based on the information in the database by themselves. They then discussed the case with each other via video-conferencing to reach an MDD diagnosis. The final diagnosis was recorded in the cloud-based database. " : interlinked by the case identification number. 
Conducting web-based MDD using the cloud-based integrated database

The database allowed users to access all three types of data for each case through the internet. We devised a model of web-based MDD using the database. A clinician, radiologist and pathologist first accessed the database to examine the case records by themselves, after which they discussed the case via video-conferencing (Arcstar Web Conferencing; NTT Communications, Tokyo, Japan) to make an MDD diagnosis (figure 1). Web-based MDD was performed for all cases.

\section{Survival analysis}

Survival data of the enrolled patients were analysed by both the institutional and MDD diagnoses. The cumulative survival rates were calculated using the Kaplan-Meier method. The log-rank test was employed to compare survival among each IIP diagnostic entity. To control for family-wise error, p-values in multiple comparisons were adjusted using Holm's method. Cox proportional hazard models were employed to identify the variables associated with a worse outcome. To compare prognostic discrimination between the institutional and MDD diagnoses, we determined the Harrell C-index for both [11, 12]. Statistical analyses were performed using JMP version 9.0 (SAS Institute, Cary, NC, USA) and R version 3.4.3 ( $\mathrm{R}$ Foundation for Statistical Computing, Vienna, Austria). A p-value $<0.05$ was considered statistically significant.

\section{Results}

\section{Date collection and development of the cloud-based integrated database}

Clinical, radiological and pathological data of 524 patients were collected from the 39 participating institutions and successfully uploaded to the respective web servers with the appropriate case identification numbers. The data were accessible and available for inspection through the internet in either English or Japanese. A login identifier and password were required to log into the database system. Supplementary figure E1 shows representative images of the user interface, including the list of enrolled cases (supplementary figure E1a and b) and the function of the "display" button. The diagnosis made at each institution and the patient's background, laboratory findings and treatment records could easily be retrieved (supplementary figures E2-E4). The HRCT and lung tissue slide images were accessed by clicking on the "DICOM" and "pathological data" buttons, respectively (supplementary figure E1c). Representative HRCT images are shown in figure $2 \mathrm{a}$. The images could be freely repositioned by the user. The whole-slide images of lung specimens at low and high magnification are shown in figure $2 \mathrm{~b}$ and $\mathrm{c}$, respectively. The Silverlight plugin (Microsoft, Redmond, WA, USA) was required to view the whole-slide images.

\section{Patients characteristics and MDD using the cloud-based integrated database}

Among 524 patients whose data were uploaded to the database, 26 had an institutional diagnosis of an ILD other than IIPs. Of the 498 patients with an institutional diagnosis of IIPs, 33 had insufficient HRCT, pathological and/or prognostic data, and were excluded from analysis. This left 465 cases with an institutional diagnosis of IIPs for analysis in this study (figure 3). Diagnoses made after the MDD were categorised according to the IPF statements [2] and the IIP classification [3] as follows: IPF, iNSIP, COP, desquamative interstitial pneumonia (DIP)/respiratory bronchiolitis-associated ILD (RB-ILD), acute interstitial pneumonia (AIP), lymphoid interstitial pneumonia (LIP), idiopathic pleuroparenchymal fibroelastosis (iPPFE), unclassifiable IIPs and other diseases (not IIPs).

The clinical characteristics of the enrolled patients are presented in table 1 . The median patient age was 65 years and 65\% were male. Mildly reduced diffusing capacity of the lung for carbon monoxide and elevated Krebs von den Lungen-6 levels were observed. The institutional and MDD diagnoses are shown in table 2. The most prevalent MDD diagnosis was IPF (43\%), followed by unclassifiable IIPs (36\%).

A chord diagram comparing institutional and MDD diagnoses is shown in figure 4. Overall, the MDD resulted in a change in a diagnosis for 219 patients (47\%). As shown in supplementary figure E5, among institutional diagnoses of IPF, 59 cases (26\%) were reclassified as unclassifiable IIPs, while 151 (67\%) were again diagnosed as IPF by the MDD. The MDD led to a decrease in the diagnosis of iNSIP from $21 \%$ to 9\%. Among institutional diagnoses of iNSIP, 42 cases (43\%) were recategorised as unclassifiable IIPs, 17 $(17 \%)$ as IPF and three (3\%) as connective tissue disease-related ILD (CTD-ILD). Unclassifiable IIPs was more common as an MDD diagnosis (36\%) than as an institutional diagnosis (20\%). One-third of patients with an MDD diagnosis of unclassifiable IIPs had an institutional diagnosis of IPF, and a quarter each of unclassifiable IIPs and iNSIP (supplementary figure E6). Major causes of unclassifiable IIPs on MDD were "multiple HRCT and/or pathological patterns that may be encountered in patients with IIP", followed by "new entity, or unusual variant of recognised entity" (supplementary figure E7). All 18 patients diagnosed with iPPFE by MDD had abnormalities with an upper or mid-lung field predominance and distinct findings of PPFE on HRCT (bilateral, upper lobe and subpleural dense consolidations with or without pleural thickening [13]). In addition, 12 of the 18 had fibrotic changes in the lower lung fields. No patient 
a)

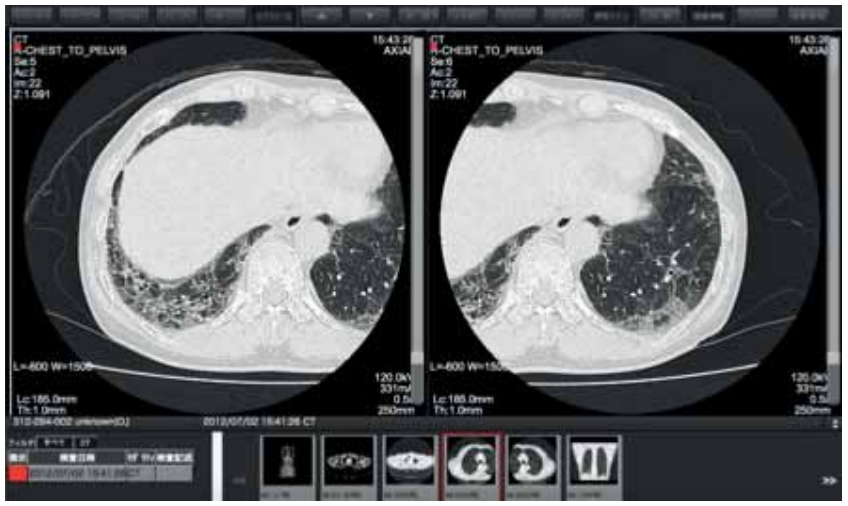

b)

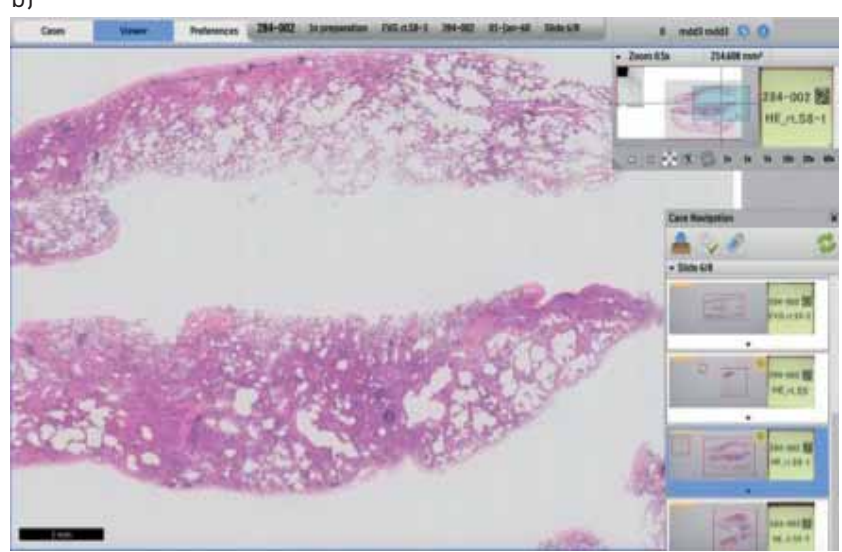

c)

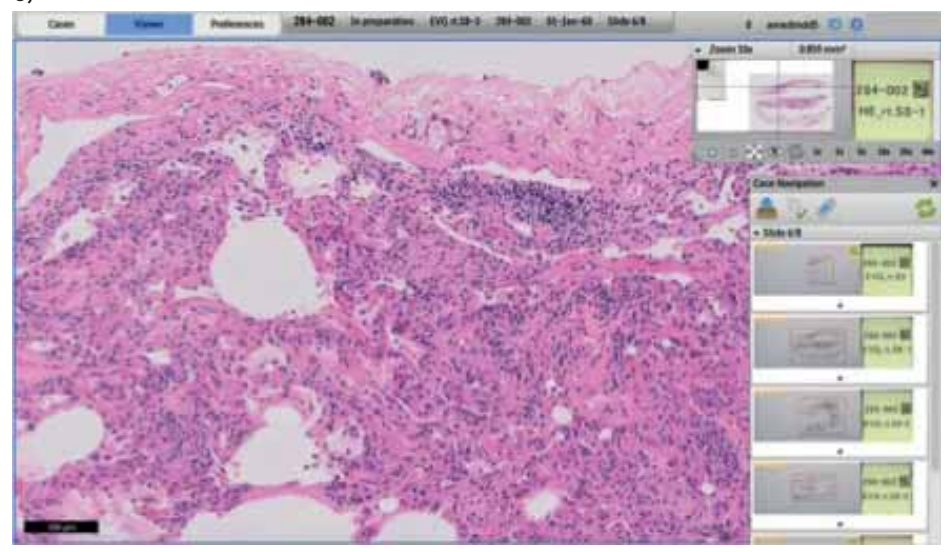

FIGURE 2 Chest high-resolution computed tomography (HRCT) and lung biopsy specimen in the cloud-based integrated database. Representative images of a) chest HRCT, and images of a whole slide of a lung biopsy specimen at b) low magnification and c) high magnification.

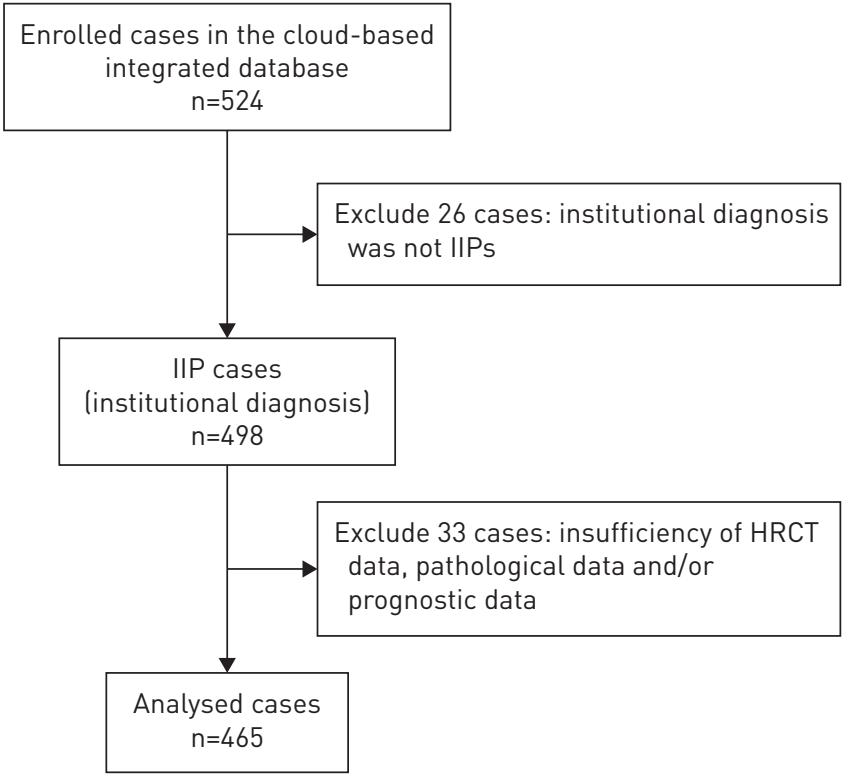

FIGURE 3 Study flowchart. IIP: idiopathic interstitial pneumonia; HRCT: high-resolution computed tomography; ILD: interstitial lung disease. Among 524 cases uploaded into the cloud-based integrated database, 26 patients had an institutional diagnosis of an ILD other than IIPs le.g. chronic hypersensitivity pneumonitis or connective tissue disease-related ILD). Of the 498 patients with IIPs as in institutional diagnosis, 33 had insufficient HRCT data, pathology data and/or prognostic data, and were excluded, leaving 465 patients with an institutional diagnosis of IIPs for analysis. 


$\begin{array}{lc}\text { TABLE } 1 \text { Patient characteristics } & \\ \text { Subjects } & 465 \\ \text { Age years } & 65(60-70) \\ \text { Sex } & 304(65) \\ \quad \text { Male } & 161(35) \\ \quad \text { Female } & 175(38) \\ \text { Never-smoker } & 82.0(69.1-93.9) \\ \text { FVC \% pred } & 84.0(73.0-95.3) \\ \text { FEV } 1 \% \text { pred } & 67.1(53.5-83.0) \\ \text { D.co \% pred } & 1054(633-1731) \\ \text { KL-6 U.mL } & -1 \\ \text { SP-D ng.mL } & 200(129-324)\end{array}$

Data are presented as $\mathrm{n}$, median (interquartile range) or $\mathrm{n}(\%)$. FVC: forced vital capacity; FEV1: forced expiratory volume in $1 \mathrm{~s}$; DLCo: diffusing capacity of the lung for carbon monoxide; KL-6: Krebs von den Lungen-6; SP-D: surfactant protein D.

was diagnosed as having AIP or LIP by MDD. 21 (5\%) patients deemed not to have IIPs were diagnosed with other diseases (chronic hypersensitivity pneumonitis (CHP) $n=12$, CTD-ILD $n=5$ and lymphoproliferative disorder $n=4)$. Interstitial pneumonia with autoimmune features (IPAF) was diagnosed in $65(14 \%)$ of the 465 enrolled cases according to IPAF criteria [14]. Of 44 patients with an MDD diagnosis of iNSIP, a substantial proportion (19 patients (43\%)) fulfilled IPAF criteria.

\section{Survival analysis comparing institutional and MDD diagnoses}

Survival curves for the patient cohort subdivided by institutional and MDD diagnoses are shown in figure $5 \mathrm{a}$ and $\mathrm{b}$, respectively. Survival analysis demonstrated that patients with IPF had a significantly worse survival than those with non-IPF for both institutional and MDD diagnoses (institutional diagnosis: $\mathrm{p}<0.0001$; MDD diagnosis: $\mathrm{p}<0.0001$ ). To examine differences in prognostic discrimination between the institutional and MDD diagnoses, we performed a log-rank test between adjacent curves (DIP/RB-ILD versus COP, COP versus iNSIP, iNSIP versus unclassifiable IIPs, unclassifiable IIPs versus IPF and IPF versus iPPFE) and corrected the five p-values using Holm's method. Among MDD diagnoses, patients with unclassifiable IIPs had a significantly poorer outcome than those with iNSIP $(p=0.034)$ and those with IPF had a poorer outcome than those with unclassifiable IIPs $(p=0.002)$ (table 3). Notably, patients with iPPFE according to the MDD diagnosis had significantly worse survival than those with IPF ( $p=0.003$ ) (table 3). The median length of survival and 5-year survival rate were 2.8 years and $23.3 \%$, respectively, for iPPFE. The prognostic significance of MDD diagnoses were evaluated using multivariate Cox proportional hazard regression analysis. Even after adjusting for age and forced vital capacity (percentage predicted), a diagnosis of unclassifiable IIPs was an independent predictor of overall mortality compared with iNSIP (supplementary table E1). Similarly, IPF was a significant predictor of poor outcome compared with unclassifiable IIPs and iPPFE was a significant predictor of poor outcome compared with IPF (supplementary table E1). In contrast to the MDD diagnosis, no significant differences were observed in survival between iNSIP and unclassifiable IIPs, unclassifiable IIPs and IPF or IPF and iPPFE based on

TABLE 2 Institutional and multidisciplinary discussion (MDD) diagnoses

Institutional diagnosis

MDD diagnosis

\begin{tabular}{lcc}
\hline IPF & $227(49)$ & $200(43)$ \\
iNSIP & $99(21)$ & $44(9)$ \\
COP & $20(4)$ & $5(1)$ \\
DIP/RB-ILD & $16(3)$ & $9(2)$ \\
LIP & $5(1)$ & 0 \\
iPPFE & $7(2)$ & $18(4)$ \\
Unclassifiable IIPs & $91(20)$ & $168(36)$ \\
Other diseases (not IIPs) & 0 & $21(5)$
\end{tabular}

Data are presented as $\mathrm{n}(\%)$. IPF: idiopathic pulmonary fibrosis; iNSIP: idiopathic nonspecific interstitial pneumonia; COP: cryptogenic organising pneumonia; DIP: desquamative interstitial pneumonia; RB-ILD: respiratory bronchiolitis-interstitial lung disease; LIP: lymphoid interstitial pneumonia; iPPFE: idiopathic pleuroparenchymal fibroelastosis; IIP: idiopathic interstitial pneumonia. 


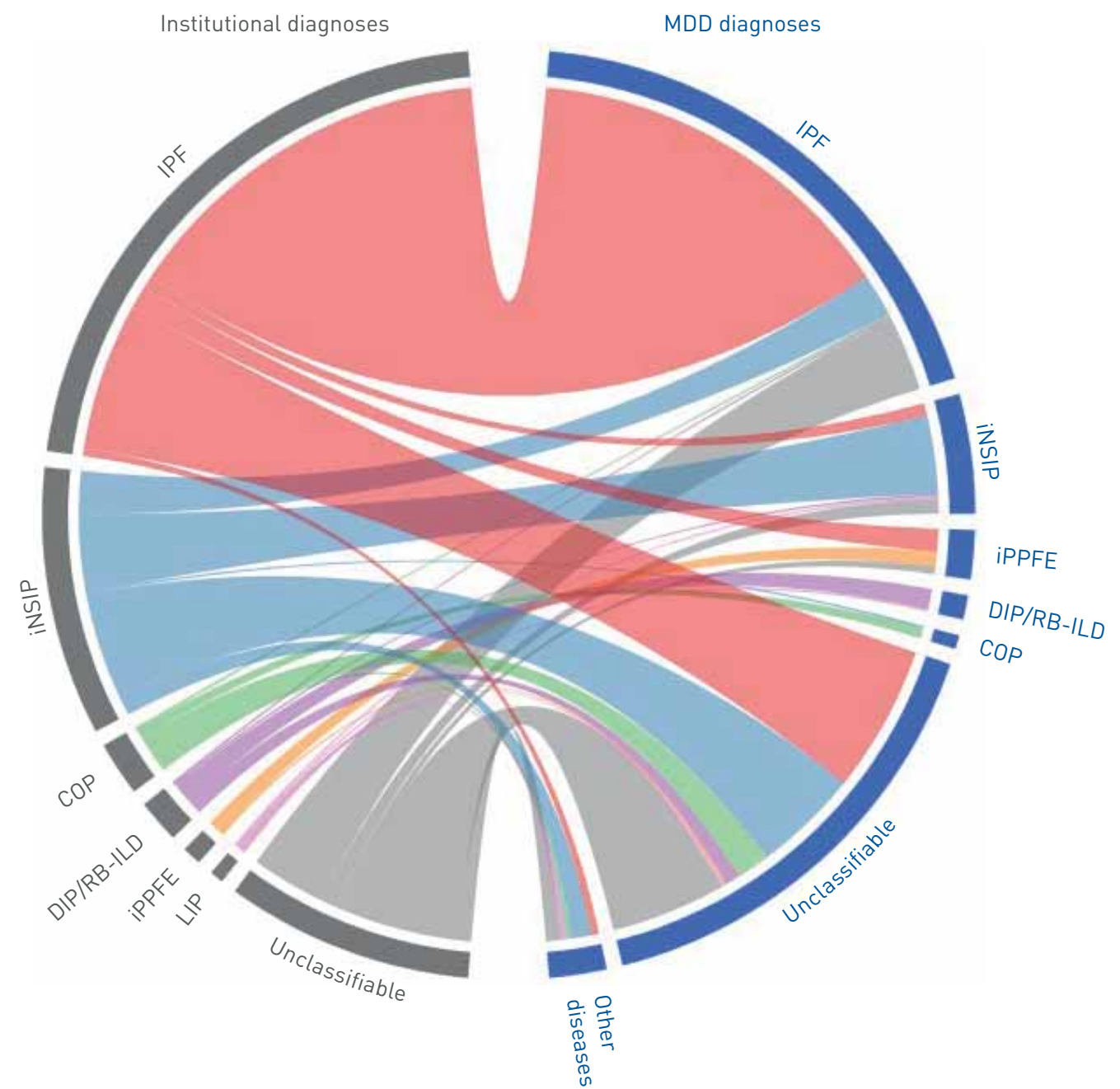

FIGURE 4 Chord diagram comparing institutional diagnoses (left) and multidisciplinary discussion (MDD) diagnoses (right). IPF: idiopathic pulmonary fibrosis; iNSIP: idiopathic nonspecific interstitial pneumonia; COP: cryptogenic organising pneumonia; DIP: desquamative interstitial pneumonia; RB-ILD: respiratory bronchiolitis-interstitial lung disease; iPPFE: pleuroparenchymal fibroelastosis; LIP: lymphoid interstitial pneumonia.

the institutional diagnosis (table 3). The prognostic discrimination between IIP disease entities was improved by the MDD diagnosis compared with the institutional diagnosis as assessed by the Harrell C-index ( 0.654 versus 0.610 , respectively). These results indicate that diagnosis by MDD was superior to institutional diagnosis in separating IIP disease entities prognostically. Survival curves for the patient cohort were subdivided by each type of specialist (pulmonologist, radiologist and pathologist) (supplementary figure E8). The Harrell C-index was 0.614 for pulmonologist diagnosis, 0.614 for radiologist diagnosis and 0.621 for pathologist diagnosis, all of which were lower than the 0.654 index for MDD diagnosis. These findings indicate that MDD with all three specialists pooling their expertise was indispensable for accurate diagnosis of biopsy-proven IIPs.

\section{Discussion}

In the present study, we report the development of a nationwide cloud-based integrated database containing clinical, radiological and pathological data of patients with IIPs, based on data from 465 biopsy-proven cases of IIPs. Specialists (pulmonary physicians, radiologists and pathologists) could readily refer to the database on the internet regardless of their physical location. Subsequently, through an online meeting, MDD diagnoses were successfully made by these specialists. Overall, MDD using this system resulted in a change in IIP diagnosis for 219 patients (47\%). Notably, the MDD diagnosis yielded better prognostic separation among iNSIP, unclassifiable IIPs, IPF and iPPFE than did the institutional diagnosis. 

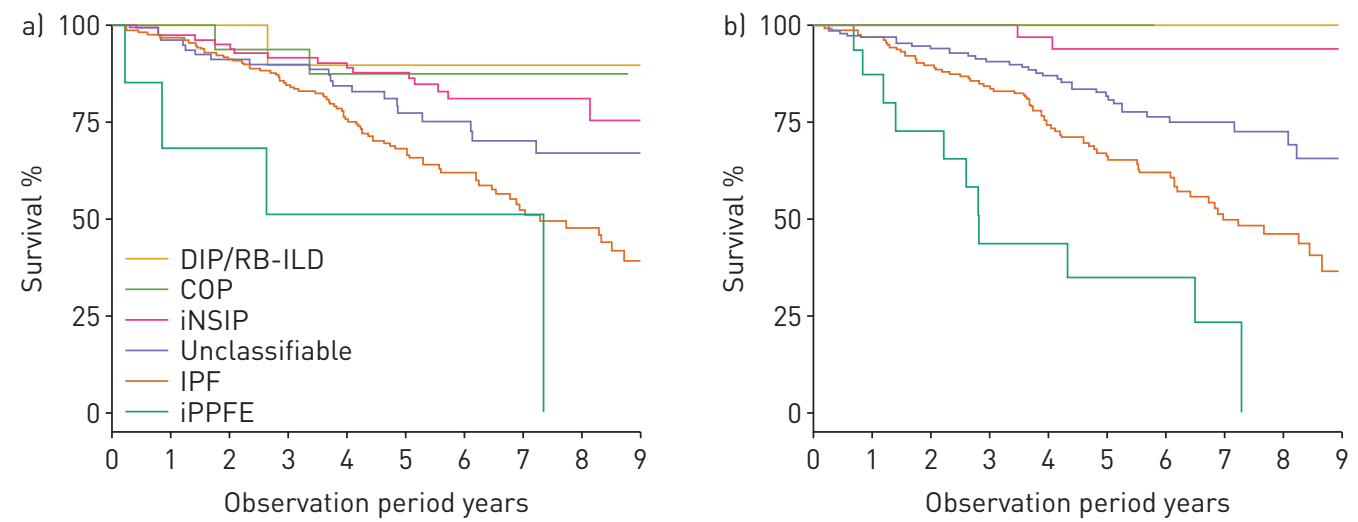

FIGURE 5 Kaplan-Meier survival curves for patients with idiopathic interstitial pneumonias in the cohort subdivided by al institutional diagnoses and b) multidisciplinary discussion (MDD) diagnoses. DIP: desquamative interstitial pneumonia; RB-ILD: respiratory bronchiolitis-interstitial lung disease; COP: cryptogenic organising pneumonia; iNSIP: idiopathic nonspecific interstitial pneumonia; IPF: idiopathic pulmonary fibrosis; iPPFE: idiopathic pleuroparenchymal fibroelastosis. Lymphoid interstitial pneumonia was excluded because no patient was diagnosed with this disease during the MDD.

The cloud-based integrated database and the web-based MDD system that we built increases the feasibility of MDD and should result in increased accuracy of diagnosis for patients with IIPs in routine practice.

The most pivotal feature of our cloud-based integrated database is the sharing of clinical, radiological and pathological data anytime and anywhere on the internet. IIPs are a diverse and challenging group of pulmonary diseases with varying prognoses and therapies. MDD is essential in determining the correct diagnosis. The guidelines for IPF [2, 4] and for IIPs [3] strongly recommend MDD for this purpose. However, MDD is not feasible in routine clinical practice. To begin with, there are a few radiologists or pathologists specialising in ILD in our country (Japan). Additionally, pulmonary physicians, radiologists and pathologists are often separated by time, geographic location and schedule. Thus, it is difficult to coordinate their timetables to participate in MDD at the same place. Our cloud-based integrated database system resolves these underlying issues in MDD by allowing patient data to be accessed easily by the specialists at their own convenience, after which they can meet by video-conferencing and come to a consensus diagnosis. With the information always available for review on the database, there is no need to gather specialists in one place or to waste time transferring data to other institutions. This cloud-based integrated database system can thus make MDD more practical in the clinic.

Another strength of our cloud-based integrated database is that, in addition to the clinical and radiological data, the pathological data were also available. Web-based access to medical images (e.g. HRCT) in the DICOM file format is widely relied upon in modern radiology departments $[15,16]$. However, few systems are available for accessing pathology images on the web. To engage in MDD on the internet, it is essential that the pathological data can be viewed in the database in the context of the clinical and radiological data. Recent advances have made it possible to create a high-resolution digital image of an entire glass slide using sophisticated digital scanning systems. In the present study, we made whole-slide images of lung

TABLE 3 Survival analysis of entities based on institutional or multidisciplinary discussion (MDD) diagnosis

Institutional diagnosis adjusted $\mathrm{p}$-value
MDD diagnosis adjusted $\mathrm{p}$-value ${ }^{\#}$

\begin{tabular}{lcc}
\hline DIP/RB-ILD versus COP & 1 & 1 \\
COP versus iNSIP & 1 & 1 \\
iNSIP versus unclassifiable IIPs & 0.511 & 0.034 \\
Unclassifiable IIPs versus IPF & 0.104 & 0.002 \\
IPF versus iPPFE & 0.450 & 0.003
\end{tabular}

DIP: desquamative interstitial pneumonia; RB-ILD: respiratory bronchiolitis-interstitial lung disease; COP: cryptogenic organising pneumonia; iNSIP: idiopathic nonspecific interstitial pneumonia; IIP: idiopathic interstitial pneumonia; IPF: idiopathic pulmonary fibrosis; iPPFE: idiopathic pleuroparenchymal fibroelastosis. \# : log-rank, adjusted using Holm's method. 
tissues from 465 patients with an institutional diagnosis of IIPs and successfully uploaded the images to the cloud-based database. This enabled the specialists to refer to them easily. Our database is the first to incorporate pathological data in the form of whole-slide images along with clinical and radiological data.

In the present study, MDD using our system changed the institutional diagnosis in 219 out of 465 patients (47\%). Importantly, this diagnostic reclassification by the MDD led to more distinct prognostic discrimination among the IIP disease entities, including iNSIP, unclassifiable IIPs, IPF and iPPFE, compared with the institutional diagnosis. Interestingly, we found that patients with an MDD diagnosis of iPPFE had a significantly worse outcome than those with IPF. This diagnosis portended a poorer prognosis than DIP/RB-ILD, COP, iNSIP or IPF. iPPFE is characterised by fibrotic thickening of the pleural and subpleural parenchyma predominantly in the upper lobes $[13,17]$, which is a rare form of IIP according to the 2013 American Thoracic Society (ATS)/European Respiratory Society (ERS) IIP guidelines [3]. A few studies have reported that 5-year survival with iPPFE was relatively poor at 30-50\% $[18,19]$. Recently, SHioy et al. [20] retrospectively analysed the characteristics of iPPFE versus IPF and found that the outcome of iPPFE was significantly worse than that of IPF. Consistent with these reports, the present study demonstrated a distinctly worse outcome of iPPFE diagnosed by MDD compared with other IIPs, including IPF. While iPPFE was less frequent than IPF in this cohort, its poor prognosis emphasises the importance of accurately diagnosing the specific type of IIPs. The number of patients diagnosed with iPPFE increased from $n=7$ by institutional diagnosis to $n=18$ by MDD diagnosis. As iPPFE was first included as an IIP in the 2013 ATS/ERS IIP guidelines [3], patients whose data had been collected before 2013 were likely to have had a different institutional diagnosis. This might partly explain the increased number of MDD iPPFE diagnoses.

In this cohort, $36 \%$ of patients had unclassifiable IIPs based on the MDD diagnosis, a relatively higher rate than that in previous studies using $\operatorname{MDD}[12,21-23]$. As all the patients enrolled in this cohort had undergone SLB, patients with typical clinical and radiological characteristics of IPF were mostly excluded. Thus, our study had some selection bias, which might be a major reason for the high frequency of unclassifiable IIPs. To date, there have been several studies of MDD for diagnosing ILDs; however, these included only small proportions of patients who had undergone SLB. The landmark study of MDD by WALSH et al. [24] included only 22 out of 70 patients (31\%) who had had a SLB. Jo et al. [25] investigated the impact of MDD on ILD diagnosis in 90 patients, but SLB had been performed for only 16 patients (18\%). Thus, the true proportion of unclassified IIP among biopsy-proven IIPs has not been clearly determined. The present study included the largest number of patients with biopsy-proven IIPs reported so far. As cohorts of patients with biopsy-proven IIPs are thought to include a considerable number of cases in which the diagnosis is difficult, we think the proportion of unclassifiable IIPs in such a cohort may be higher than in IIP cohorts that include cases where a biopsy was thought to be unnecessary for diagnosis. Survival with unclassifiable IIPs has been reported to be intermediate between that of IPF and non-IPF [26]. Consistent with that conclusion, we found that survival of unclassifiable IIPs was significantly better than that of IPF, but worse than that of iNSIP. However, unclassifiable IIPs have substantial heterogeneity in their clinical course, response to treatment and outcome [21, 22, 27]. In addition, there have been wide variations and inconsistencies in the terminology and definitions of unclassifiable IIPs. Further investigation will be required to better understand this group of disorders.

Our cohort did not reflect true populations of patients with IIPs in the real world, because all patients included underwent SLB. Accordingly, the observations of the present study, such as the proportion of each category of IIP diagnosis, may not be applicable to general populations with IIPs. It is of course very important in the clinical setting to differentiate IIPs from ILDs with a known cause, such as CHP or CTD-ILD. Therefore, it will be important to evaluate the performance of MDD diagnosis for a wider range of ILDs. However, the present study, which was funded by the Japanese Ministry of Health, formed a part of the Practical Research Project for Rare Intractable Diseases from the Japan Agency for Medical Research and Development. The definition of rare diseases included IIPs, but excluded other entities such as CHP or CTD-ILD. Our nationwide database for this study was therefore designed to include only patients with IIPs. In this context, the present study clearly indicates that our MDD system performed well in our cohort with good feasibility. The web-based MDD system can easily be applicable to other ILDs. Thus, this system we built could be a promising platform to enable MDD for a variety of ILDs other than IIPs. In the clinical setting, MDD is also performed in cases of ILD without SLB. To further explore this concept, we are currently planning a prospective cohort study including ILDs other than biopsy-proven IIPs. In addition, the digitised clinical, radiological and pathological data of patients with IIPs diagnosed by MDD in our database may be a valuable resource for developing an artificial intelligence-based multimodal diagnostic system for ILD.

There are some limitations to the present study. We successfully carried out web-based MDD using our integrated database. However, a direct data-upload system for the three types of data from each institution 
has not yet been established. When enrolling new cases of IIPs in the real world, a convenient system for data upload to the web will be necessary. We are now planning to develop such a system. Second, disease behaviour was not considered in making the MDD diagnosis, because the clinical and radiological data considered during the MDD were only current up to the time of the SLB, and no further information (e.g. sequential clinical course) was available in the database, which might partly account for a relatively higher incidence of unclassifiable IIPs. Third, MDD was conducted by pulmonologists, radiologists and pathologists. Neither rheumatologists nor occupational medicine specialists participated in MDDs in this study. Fourth, the quality of the history and physical examination available in the database depended on the experience of each patient's attending physician in managing ILD and might have varied. Lastly, this was a retrospective cohort study; however, given our findings of the high diagnostic performance and feasibility of our web-based MDD system, a prospective cohort study using this system and including a larger number of patients with ILD is currently being undertaken in Japan.

In conclusion, we developed a nationwide cloud-based integrated database that contains clinical, radiological and pathological data of patients with biopsy-proven IIPs. Using this database, we also built a web-based MDD system that enabled successful conduct of web-based MDD for a large number of patients with IIPs. This database and web-based MDD system make the performance of MDD more feasible in clinical practice, which could increase the accuracy of diagnosis for patients with IIPs.

Acknowledgements: We greatly appreciate the investigators in the participating institutions for their support in data collection (Asahikawa Medical University Respiratory Center, Asahikawa: Yoshinobu Ohsaki; First Division, Dept of Internal Medicine, Obihiro Kosei General Hospital, Obihiro: Kei Takamura; Division of Pulmonary Medicine, Dept of Medicine, Jichi Medical University, Shimotsuke: Masashi Bando; Dept of Respiratory Medicine, Respiratory Center, Toranomon Hospital, Tokyo: Atsushi Miyamato; Division of Respiratory Diseases, Dept of Internal Medicine, Jikei University School of Medicine, Tokyo: Takanori Numata; Dept of Respiratory Medicine, Juntendo University Graduate School of Medicine, Tokyo: Hiroaki Ihara; Dept of Respiratory Medicine, Japanese Red Cross Medical Center, Tokyo: Nobuyasu Awano; Division of Respiratory Medicine, Dept of Internal Medicine, Toho University Ohashi Medical Center, Tokyo: Toru Yamagishi; Dept of Respiratory Medicine, Nagano Red Cross Hospital, Nagano: Manabu Yamamoto; Respiratory Division, Nagano Municipal Hospital, Nagano: Fumiaki Yoshiike; First Dept of Internal Medicine, Shinshu University School of Medicine, Matsumoto: Hiroshi Yamamoto; Dept of Respiratory Medicine, Shizuoka General Hospital, Shizuoka: Toshihiro Shirai; Dept of Pulmonary Medicine, Seirei Hamamatsu General Hospital, Hamamatsu: Hidenori Nakamura; Dept of Respiratory Medicine, Hamamatsu Rosai Hospital, Hamamatsu: Mikio Toyoshima; Dept of Respiratory Medicine, Respiratory Disease Center, Seirei Mikatahara General Hospital, Hamamatsu: Koshi Yokomura; Division of Respiratory Medicine, Iwata City Hospital, Iwata: Shiro Imokawa; Dept of Respiratory Medicine, Fujita Health University School of Medicine, Toyoake: Tomoyuki Minezawa; Dept of Respiratory Medicine, Komaki Municipal Hospital, Komaki: Eiji Kojima; Dept of Respiratory Medicine, Ogaki Municipal Hospital, Ogaki: Akira Shiraki; Dept of Respiratory Medicine, Izumiotsu Municipal Hospital, Izumiotsu: Naho Nomura; Dept of Respiratory Medicine, Kobe City Medical Center General Hospital, Kobe: Keisuke Tomii; Dept of Respiratory Medicine, Kobe City Medical Center West Hospital, Kobe: Hiromi Tomioka; Dept of Respiratory Medicine and Allergology, Kindai University, Faculty of Medicine, Osaka: Osamu Nishiyama; Dept of Respiratory Medicine, Kurashiki Central Hospital, Kurashiki: Machiko Arita; Dept of Respiratory Medicine, National Hospital Organization Yamaguchi-Ube Medical Center, Ube: Keiji Oishi; Dept of Respiratory Medicine, University of Occupational and Environmental Health, Kitakyushu: Kazuhiro Yatera; Dept of Respiratory Medicine, National Hospital Organization, Fukuoka National Hospital, Fukuoka: Hiroko Nogami; Division of Respirology, Neurology and Rheumatology, Dept of Internal Medicine, Kurume University School of Medicine, Kurume: Masaki Okamoto; Dept of Respiratory Medicine and Infectious Diseases, Oita University Faculty of Medicine, Yufu: Masaru Ando; Dept of Respiratory Medicine, Saiseikai Kumamoto Hospital, Kumamoto: Yuko Yasuda; Division of Pulmonary Medicine, National Hospital Organization Okinawa National Hospital, Okinawa: Isoko Owan; Dept of Respiratory Medicine, Okinawa Chubu Hospital, Okinawa: Tomoo Kishaba). We thank Hideki Okamura and Masayuki Hayashi (Philips Japan, Ltd, Tokyo, Japan) for their expert technical assistance.

Author contributions: T. Fujisawa: conception and design of the work, acquisition, analysis and interpretation of data, and manuscript writing; K. Mori: acquisition and analysis of data, statistical analysis; M. Mikamo: acquisition and analysis of data; T. Ohno: conception and design of the work; K. Kataoka, C. Sugimoto, H. Kitamura, N. Enomoto, R. Egashira, H. Sumikawa, T. Iwasawa, S. Matsushita, H. Sugiura, M. Hashisako, T. Tanaka, Y. Terasaki, S. Kunugi and M. Kitani: analysis of data; R. Okuda, Y. Horiike, Y. Enomoto, H. Yasui, H. Hozumi, Y. Suzuki and Y. Nakamura: acquisition of data; J. Fukuoka, T. Johkoh, Y. Kondoh, T. Ogura, Y. Inoue, Y. Hasegawa and N. Inase: conception and design of the work; S. Homma: conception and design of the work, and administrative support; T. Suda: conception and design of the work, acquisition, analysis and interpretation of data, administrative support, and manuscript writing. All authors reviewed the manuscript.

Conflict of interest: T. Fujisawa has nothing to disclose. K. Mori has nothing to disclose. M. Mikamo has nothing to disclose. T. Ohno has nothing to disclose. K. Kataoka has nothing to disclose. C. Sugimoto has nothing to disclose. H. Kitamura has nothing to disclose. N. Enomoto has nothing to disclose. R. Egashira has nothing to disclose. H. Sumikawa has nothing to disclose. T. Iwasawa has nothing to disclose. S. Matsushita has nothing to disclose. H. Sugiura receives honoraria for consultancy services from Ono Pharmaceutical Co., Ltd, Bristol-Myers Squibb KK and MSD KK. M. Hashisako has nothing to disclose. T. Tanaka has nothing to disclose. Y. Terasaki has nothing to disclose. S. Kunugi has nothing to disclose. M. Kitani has nothing to disclose. R. Okuda has nothing to disclose. Y. Horiike has nothing to disclose. Y. Enomoto has nothing to disclose. H. Yasui has nothing to disclose. H. Hozumi has nothing to disclose. Y. Suzuki has nothing to disclose. Y. Nakamura has nothing to disclose. J. Fukuoka has nothing to disclose. T. Johkoh has 
nothing to disclose. Y. Kondoh has nothing to disclose. T. Ogura has nothing to disclose. Y. Inoue reports fees for advisory board work and lecturing from Boehringer Ingelheim, outside the submitted work. Y. Hasegawa has nothing to disclose. $\mathrm{N}$. Inase has nothing to disclose. S. Homma has nothing to disclose. T. Suda has nothing to disclose.

Support statement: This research is supported by the Practical Research Project for Rare Intractable Diseases from the Japan Agency for Medical Research and Development, and Grant-in-Aid for Diffuse Lung Diseases Research Group from the Japanese Ministry of Health, Labour and Welfare. Funding information for this article has been deposited with the Crossref Funder Registry.

\section{References}

1 American Thoracic Society, European Respiratory Society. American Thoracic Society/European Respiratory Society international multidisciplinary consensus classification of the idiopathic interstitial pneumonias. Am J Respir Crit Care Med 2002; 165: 277-304.

2 Raghu G, Collard HR, Egan JJ, et al. An official ATS/ERS/JRS/ALAT statement: idiopathic pulmonary fibrosis: evidence-based guidelines for diagnosis and management. Am J Respir Crit Care Med 2011; 183: 788-824.

3 Travis WD, Costabel U, Hansell DM, et al. An official American Thoracic Society/European Respiratory Society statement: update of the international multidisciplinary classification of the idiopathic interstitial pneumonias. Am J Respir Crit Care Med 2013; 188: 733-748.

4 Raghu G, Remy-Jardin M, Myers JL, et al. Diagnosis of idiopathic pulmonary fibrosis. An official ATS/ERS/JRS/ ALAT clinical practice guideline. Am J Respir Crit Care Med 2018; 198: e44-e68.

5 Bjoraker JA, Ryu JH, Edwin MK, et al. Prognostic significance of histopathologic subsets in idiopathic pulmonary fibrosis. Am J Respir Crit Care Med 1998; 157: 199-203.

6 Wuyts WA, Antoniou KM, Borensztajn K, et al. Combination therapy: the future of management for idiopathic pulmonary fibrosis? Lancet Respir Med 2014; 2: 933-942.

7 Martinez FJ, Chisholm A, Collard HR, et al. The diagnosis of idiopathic pulmonary fibrosis: current and future approaches. Lancet Respir Med 2017; 5: 61-71.

$8 \quad$ Lederer DJ, Martinez FJ. Idiopathic pulmonary fibrosis. N Engl J Med 2018; 378: 1811-1823.

9 Flaherty KR, King TE Jr, Raghu G, et al. Idiopathic interstitial pneumonia: what is the effect of a multidisciplinary approach to diagnosis? Am J Respir Crit Care Med 2004; 170: 904-910.

10 Thomeer M, Demedts M, Behr J, et al. Multidisciplinary interobserver agreement in the diagnosis of idiopathic pulmonary fibrosis. Eur Respir J 2008; 31: 585-591.

11 Harrell FE Jr, Califf RM, Pryor DB, et al. Evaluating the yield of medical tests. JAMA 1982; 247: 2543-2546.

12 De Sadeleer LJ, Meert C, Yserbyt J, et al. Diagnostic ability of a dynamic multidisciplinary discussion in interstitial lung diseases: a retrospective observational study of 938 cases. Chest 2018; 153: 1416-1423.

13 Reddy TL, Tominaga M, Hansell DM, et al. Pleuroparenchymal fibroelastosis: a spectrum of histopathological and imaging phenotypes. Eur Respir J 2012; 40: 377-385.

14 Fischer A, Antoniou KM, Brown KK, et al. An official European Respiratory Society/American Thoracic Society research statement: interstitial pneumonia with autoimmune features. Eur Respir J 2015; 46: 976-987.

15 Arnold CW, Bui AA, Morioka C, et al. Informatics in radiology: a prototype web-based reporting system for onsite-offsite clinician communication. Radiographics 2007; 27: 1201-1211.

16 Shen $\mathrm{H}, \mathrm{Ma} \mathrm{D}$, Zhao Y, et al. MIAPS: a web-based system for remotely accessing and presenting medical images. Comput Methods Programs Biomed 2014; 113: 266-283.

17 Kusagaya H, Nakamura Y, Kono $\mathrm{M}$, et al. Idiopathic pleuroparenchymal fibroelastosis: consideration of a clinicopathological entity in a series of Japanese patients. BMC Pulm Med 2012; 12 : 72.

18 Enomoto Y, Nakamura Y, Satake Y, et al. Clinical diagnosis of idiopathic pleuroparenchymal fibroelastosis: a retrospective multicenter study. Respir Med 2017; 133: 1-5.

19 Ishii $\mathrm{H}$, Watanabe $\mathrm{K}$, Kushima $\mathrm{H}$, et al. Pleuroparenchymal fibroelastosis diagnosed by multidisciplinary discussions in Japan. Respir Med 2018; 141: 190-197.

20 Shioya M, Otsuka M, Yamada G, et al. Poorer prognosis of idiopathic pleuroparenchymal fibroelastosis compared with idiopathic pulmonary fibrosis in advanced stage. Can Respir J 2018; 2018: 6043053

21 Ryerson CJ, Urbania TH, Richeldi L, et al. Prevalence and prognosis of unclassifiable interstitial lung disease. Eur Respir J 2013; 42: 750-757.

22 Guler SA, Ellison K, Algamdi M, et al. Heterogeneity in unclassifiable interstitial lung disease. A systematic review and meta-analysis. Ann Am Thorac Soc 2018; 15: 854-863.

23 Chaudhuri N, Spencer L, Greaves M, et al. A review of the multidisciplinary diagnosis of interstitial lung diseases: a retrospective analysis in a single UK specialist centre. J Clin Med 2016; 5: E66.

24 Walsh SL, Wells AU, Desai SR, et al. Multicentre evaluation of multidisciplinary team meeting agreement on diagnosis in diffuse parenchymal lung disease: a case-cohort study. Lancet Respir Med 2016; 4: 557-565.

25 Jo HE, Glaspole IN, Levin KC, et al. Clinical impact of the interstitial lung disease multidisciplinary service. Respirology 2016; 21: 1438-1444.

26 Hyldgaard C, Bendstrup E, Wells AU, et al. Unclassifiable interstitial lung diseases: clinical characteristics and survival. Respirology 2017; 22: 494-500.

27 Guler SA, Ryerson CJ. Unclassifiable interstitial lung disease: from phenotyping to possible treatments. Curr Opin Pulm Med 2018; 24: 461-468. 